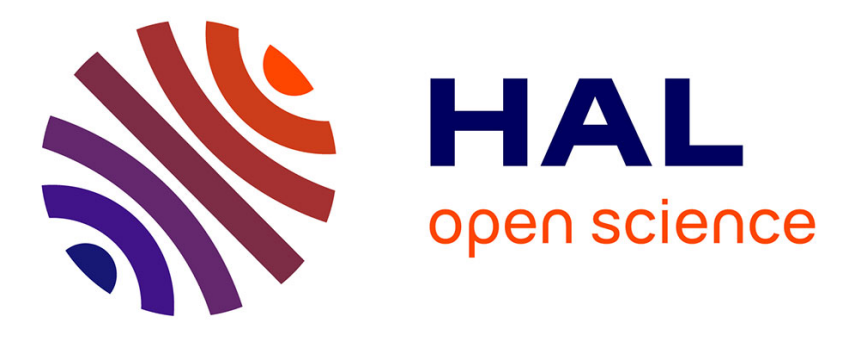

\title{
Periodic orbits in planar linear systems with input saturation
}

Thomas Lathuiliere, Giorgio Valmorbida, Elena Panteley

\section{To cite this version:}

Thomas Lathuiliere, Giorgio Valmorbida, Elena Panteley. Periodic orbits in planar linear systems with input saturation. IEEE Control Systems Letters, 2018, 2 (3), pp.435-440. 10.1109/LCSYS.2018.2841807 . hal-01811047

\section{HAL Id: hal-01811047 https://hal.science/hal-01811047}

Submitted on 9 Jan 2019

HAL is a multi-disciplinary open access archive for the deposit and dissemination of scientific research documents, whether they are published or not. The documents may come from teaching and research institutions in France or abroad, or from public or private research centers.
L'archive ouverte pluridisciplinaire $\mathbf{H A L}$, est destinée au dépôt et à la diffusion de documents scientifiques de niveau recherche, publiés ou non, émanant des établissements d'enseignement et de recherche français ou étrangers, des laboratoires publics ou privés. 


\title{
Periodic Orbits in planar linear systems with input saturation
}

\author{
Thomas Lathuilière $^{1}$ and Giorgio Valmorbida ${ }^{1}$ and Elena Panteley ${ }^{1}$
}

\begin{abstract}
We present sufficient conditions for the existence of periodic orbits of saturating planar systems. We characterize inner and outer sets bounding the periodic orbits. A method to build these bounds, based on the solution to a convex optimization problem, is proposed and illustrated with numerical examples.
\end{abstract}

Index Terms - Stability of nonlinear systems, Constrained Control

\section{INTRODUCTION}

A NY practical feedback system presents constraints that can be physical, technological or imposed by safety requirements [3]. It is thus mandatory to incorporate these constraints in the model of the system for the closed-loop analysis and design. In a large number of cases, the constraints are magnitude saturations.

Input saturation is at the origin of nonlinear phenomena such as multiple isolated equilibria or isolated periodic trajectories which might occur even in systems of low dimension. An example of limit cycles appearing in planar saturating systems is considered in [3, Chapters 4, 6].

Over the last few decades, several Lyapunov based techniques for analysis and design of systems with input nonlinearities such as saturation [10], [4], quantization [2], backlash [9] and other hysteresis functions [7] have been proposed. Importantly, these systematic approaches are often associated to numerical formulations for the computation of feedback gains. Nonetheless, in most cases, such control design techniques consider only quadratic Lyapunov functions.

On the other hand, analysis and design of periodic trajectories in linear systems with deadzone and saturation on the input has yet to be explored. In this context, a challenging question yet to be answered is: "Is it possible to characterize exactly the periodic trajectories of planar saturating systems as a level set of a function?". Also, in case the system generates periodic trajectory, it is of interest to know its amplitude and frequency.

\footnotetext{
${ }^{1}$ Thomas Lathuilière, Giorgio Valmorbida and Elena Panteley are with Laboratoire des Signaux et Systèmes, CentraleSupélec, CNRS, Univ. Paris-Sud, Université Paris-Saclay, 3 Rue Joliot Curie, Gif-sur-Yvette 91192, France. Elena Panteley is also with ITMO University, Kronverkskiy av. 49, St Petersburg, 197101, Russia. Giorgio Valmorbida is also with Inria, projet DISCO. \{thomas.lathuiliere, giorgio.valmorbida, elena.panteley\}@12s.centralesupelec.fr. This work was supported by iCODE - Institut pour le Controle et la décision de l'Idex Paris-Saclay, by L2S - Projet Jeunes Chercheurs and by the Government of Russian Federation (grant 074-U01).
}

This paper presents a first step towards the characterization of periodic trajectories of saturating linear systems. Namely, we set conditions on the data of planar saturating systems for the existence of trajectories between an attractive set and a repulsive one containing the origin. We pay a particular attention to the existence of periodic trajectories. We then propose an optimization based method to obtain two ellipsoids defining a ring that contain periodic trajectories of the system. Moreover the proposed numerical methods does not rely on sector conditions as the nonlinear analysis in [4], [2], [9], [7] in which only the convergence to an invariant set is guaranteed. Furthermore here we show the existence of a periodic orbit by ruling out the existence of equilibrium points other than the origin.

In Section II we present the class of saturating systems studied in this paper. We define a suitable piece-wise affine representation of the system and quadratic functions used for the statement of the main result. In Section III, we show how to build a set in which periodic trajectories of the system will be confined. In Section IV we present algorithms to compute these sets.

Notation. We use $M_{(i, j)}$ to denote the $(i, j)$ entry of a matrix $M$. We denote by $\Phi\left(t, x_{0}\right)$ the solution to a dynamical system initiated from the point $x_{0}$ at time $t=0$. We also denote the set of positive definite (negative definite) $\mathbb{S}_{>0}=\left\{M \in \mathbb{R}^{2 \times 2} \mid M=M^{\top}, M>0\right\}$ $\left(\mathbb{S}_{<0}=\left\{M \in \mathbb{R}^{2 \times 2} \mid M=M^{\top}, M<0\right\}\right)$ and positive semi-definite (negative semi-definite) matrices, $\mathbb{S}_{\geq 0}=\left\{M \in \mathbb{R}^{2 \times 2} \mid M=M^{\top}, M \geq 0\right\}\left(\mathbb{S}_{\leq 0}=\{M \in\right.$ $\left.\left.\mathbb{R}^{\overline{2} \times 2} \mid M=M^{\top}, M \leq 0\right\}\right)$. With $P \in \mathbb{S}_{\geq 0}$, we denote the ellipsoidal set $\mathcal{E}(P, \alpha)=\left\{x \in \mathbb{R}^{2} \mid x^{\top} \bar{P} x \leq \alpha\right\}$. The set $\operatorname{Conv}(\mathcal{A})$ is the convex hull of set $\mathcal{A}$ and $\partial \mathcal{A}$ is the boundary of set $\mathcal{A}$.

\section{Preliminaries}

Consider the class of planar linear saturating systems

$$
\dot{\bar{x}}=\bar{A} \bar{x}+\bar{B} \operatorname{sat}(\bar{K} \bar{x})
$$

with $\bar{x} \in \mathbb{R}^{2}, \bar{A} \in \mathbb{R}^{2 \times 2}, \bar{B} \in \mathbb{R}^{2 \times 1}, \bar{K} \in \mathbb{R}^{1 \times 2}$ and the unit saturation function defined by $\operatorname{sat}\left(u_{c}\right)=$ $\operatorname{sign}\left(u_{c}\right) \min \left(\left|u_{c}\right|, 1\right)$. With the coordinate transformation $x=T \bar{x}$, with

$$
T:=\frac{1}{\left(\bar{K} \bar{K}^{\top}\right)}\left[\begin{array}{ll}
\bar{K}^{\top} & \bar{K}^{\perp}
\end{array}\right]
$$

and $\bar{K}^{\perp} \in \mathbb{R}^{2 \times 1}$ satisfying $\bar{K} \bar{K}^{\perp}=0$, system (1) becomes

$$
\dot{x}=A x+B \operatorname{sat}(K x)
$$


with $A=T^{-1} \bar{A} T, B=T^{-1} \bar{B}$ and $K=\left[\begin{array}{ll}1 & 0\end{array}\right]$.

Remark 2.1: Note that the above system model also accommodates systems with deadzone nonlinearity by using the identity $\operatorname{sat}\left(u_{c}\right)+\mathrm{dz}\left(u_{c}\right)=u_{c}$.

With the definition of the saturation function, the above system can be put in the following piecewise affine form

$\dot{x}= \begin{cases}A x-B & \text { if } x \in \mathcal{R}_{L}:=\left\{x \in \mathbb{R}^{2} \mid x_{1}<-1\right\} \\ A_{C L} x & \text { if } x \in \mathcal{R}_{C}:=\left\{x \in \mathbb{R}^{2} \mid-1 \leq x_{1} \leq 1\right\} \\ A x+B & \text { if } x \in \mathcal{R}_{R}:=\left\{x \in \mathbb{R}^{2} \mid 1<x_{1}\right\}\end{cases}$

with $A_{C L}=A+B\left[\begin{array}{ll}1 & 0\end{array}\right]$. We also introduce the sets defining the boundaries between $\mathcal{R}_{C}, \mathcal{R}_{L}$ and $\mathcal{R}_{R}$ that is $\mathcal{D}_{1}:=\left\{x \in \mathbb{R}^{2}, x_{1}=1\right\}, \mathcal{D}_{-1}:=\left\{x \in \mathbb{R}^{2}, x_{1}=-1\right\}$

We consider the following assumption

Assumption 2.1: $A$ and $-A_{C L}$ are Hurwitz matrices.

Note that the above assumption does not require either the eigenvalues of $A$ or $A_{C L}$ to be complex conjugate. Note that if the pair $(\bar{A}, \bar{B})$ is controllable (hence pair $(A, B)$ is controllable) and $\bar{A}$ is Hurwitz it is always possible to obtain $\bar{K}$ such that $\bar{A}+\bar{B} \bar{K}$ gives $A_{C L}$ such that the pair $A$ and $A_{C L}$ satisfy Assumption 2.1. The lemma below concerns the equilibria of (3).

Lemma 2.1: Under Assumption 2.1, the origin is the only equilibrium point of system (3).

Proof: Denote $A=\left[\begin{array}{ll}a_{11} & a_{12} \\ a_{21} & a_{22}\end{array}\right], B=\left[\begin{array}{l}b_{1} \\ b_{2}\end{array}\right]$ yielding $A_{C L}=\left[\begin{array}{ll}a_{11}+b_{1} & a_{12} \\ a_{21}+b_{2} & a_{22}\end{array}\right]$.

From Assumption 2.1, $\operatorname{trace}(A)<0, \operatorname{det}(A)>0$ and $\operatorname{trace}\left(A_{C L}\right)>0, \operatorname{det}\left(A_{C L}\right)>0$, that is

$$
\left\{\begin{array}{l}
a_{11}+a_{22}<0 \\
a_{11} a_{22}-a_{21} a_{12}>0
\end{array}, \quad\left\{\begin{array}{l}
a_{11}+b_{1}+a_{22}>0 \\
\left(a_{11}+b_{1}\right) a_{22} \\
-\left(a_{21}+b_{2}\right) a_{12}>0 .
\end{array}\right.\right.
$$

From (3) the possible equilibria are $x_{L}=A^{-1} B$ if $x_{L} \in$ $\mathcal{R}_{L}, x_{C}=0$ and $x_{R}=-A^{-1} B$ if $x_{R} \in \mathcal{R}_{R}$.

Note that $x_{L} \in \mathcal{R}_{L} \Leftrightarrow K A^{-1} B<-1$ and $x_{R} \in \mathcal{R}_{R} \Leftrightarrow$ $-K A^{-1} B>1$. Let us show that $-K A^{-1} B>1$ can not hold. We have

$$
K A^{-1} B=\frac{a_{22} b_{1}-a_{12} b_{2}}{\operatorname{det}(A)} .
$$

Suppose that $-K A^{-1} B>1$ holds, that is $\operatorname{det}(A)+a_{22} b_{1}-$ $a_{12} b_{2}<0$ and $\left(a_{11}+b_{1}\right) a_{22}-\left(a_{21}+b_{2}\right) a_{12}<0$ which contradicts (4). Thus, $-K A^{-1} B>1$ can not hold. It follows that $K A^{-1} B<-1$ can not hold either and we conclude that the origin is the only equilibrium point of (3).

Under Assumption 2.1, given $Q_{1} \in \mathbb{S}_{<0}$ and $Q_{2_{C L}} \in$ $\mathbb{S}_{>0}, \exists P_{1}, P_{2} \in \mathbb{S}_{>0}$ satisfying the Lyapunov equations

$$
\begin{aligned}
A^{\top} P_{1}+P_{1} A & =Q_{1} \\
A_{C L}^{\top} P_{2}+P_{2} A_{C L} & =Q_{2_{C L}} .
\end{aligned}
$$

With $P_{1}, P_{2}$ satisfying the above equations, define

$$
\begin{aligned}
Q_{1_{C L}} & :=A_{C L}^{\top} P_{1}+P_{1} A_{C L} \\
Q_{2} & :=A^{\top} P_{2}+P_{2} A .
\end{aligned}
$$

Lemma 2.2: $Q_{2}$ and $Q_{1_{C L}}$ are not sign definite and are invertible.

Proof: We have $x^{\top} Q_{i_{C L}} x=x^{\top} Q_{i} x+2 x_{1} B^{\top} P_{i} x$, for $i \in\{1,2\}$, take $x=\left[\begin{array}{l}0 \\ 1\end{array}\right]$ to obtain

$$
\left[\begin{array}{ll}
0 & 1
\end{array}\right] Q_{i_{C L}}\left[\begin{array}{l}
0 \\
1
\end{array}\right]=\left[\begin{array}{ll}
0 & 1
\end{array}\right] Q_{i}\left[\begin{array}{l}
0 \\
1
\end{array}\right]=Q_{i(2,2)} .
$$

From (7), since $Q_{2_{C L}} \in \mathbb{S}_{>0}$ we have that $Q_{2(2,2)}>0$, hence $Q_{2} \notin \mathbb{S}_{\leq 0}$. By Assumption $2.1 A$ is Hurwitz, thus since $P_{2}>0, Q_{2} \notin \mathbb{S}_{\geq 0}$. To prove this claim, suppose $Q_{2} \in \mathbb{S}_{\geq 0}$. Consider a quadratic function $V(x)=x^{T} P_{2} x$, with $P_{2}$ in (5b). Differentiating $V(x)$ along the trajectories of $\dot{x}=A x$ and using (6b) gives $\dot{V}(x)=x^{T} Q_{2} x \geq 0$, which implies that $V(x(t)) \geq V(x(0)) \forall t$, therefore contradicting $\lim _{t \rightarrow \infty} x(t)=0$ for $\dot{x}=A x$ which stems from the fact that $A$ is Hurwitz. We thus conclude that $Q_{2}$ can not be positive semi-definite. Since $Q_{2} \notin \mathbb{S}_{\geq 0}, Q_{2} \notin \mathbb{S}_{\leq 0}$ and $n=$ 2 , both eigenvalues of $Q_{2}$ are not zero ( $Q_{2}^{-1}$ exists), and have opposite sign $\left(Q_{2}\right.$ is not sign definite).

The proof is similar for $Q_{1_{C L}}$ and is thus omitted.

Let us now define quadratic functions $V_{i}(x):=x^{\top} P_{i} x$, $i \in\{1,2\}$ functions associated to the solutions $P_{1}, P_{2}$ of (5) and introduce $\dot{V}_{i L}, \dot{V}_{i C}, \dot{V}_{i R}: \mathbb{R}^{2} \rightarrow \mathbb{R}$

$$
\begin{aligned}
& \dot{V}_{i L}(x):=x^{\top} Q_{i} x-2 x^{\top} P_{i} B \\
& \dot{V}_{i C}(x):=x^{\top} Q_{i_{C L}} x \\
& \dot{V}_{i R}(x):=x^{\top} Q_{i} x+2 x^{\top} P_{i} B .
\end{aligned}
$$

Using these notations, the time-derivative of the functions $V_{i}$ along trajectories of (3) is given by

$$
\dot{V}_{i}(x)= \begin{cases}2 x^{\top} P_{i}(A x-B)=\dot{V}_{i L}(x) & \text { if } x \in \mathcal{R}_{L} \\ 2 x^{\top} P_{i} A_{C L} x=\dot{V}_{i C}(x) & \text { if } x \in \mathcal{R}_{C} \\ 2 x^{\top} P_{i}(A x+B)=\dot{V}_{i R}(x) & \text { if } x \in \mathcal{R}_{R}\end{cases}
$$

Note that $\dot{V}_{i} \in \mathcal{C}^{0}$ since the vector field (3) is continuous.

Now define the sets $\mathcal{Z}_{i}:=\left\{x \in \mathbb{R}^{2} \mid \dot{V}_{i}(x)=0\right\}, i \in$ $\{1,2\}$ and introduce

$$
\begin{aligned}
& \mathcal{Z}_{i L}:=\left\{x \in \mathbb{R}^{2} \mid \dot{V}_{i L}(x)=0\right\} \\
& \mathcal{Z}_{i C}:=\left\{x \in \mathbb{R}^{2} \mid \dot{V}_{i C}(x)=0\right\} \\
& \mathcal{Z}_{i R}:=\left\{x \in \mathbb{R}^{2} \mid \dot{V}_{i R}(x)=0\right\}
\end{aligned}
$$

such that with the partition $\left\{\mathcal{R}_{L}, \mathcal{R}_{C}, \mathcal{R}_{R}\right\}$ of $\mathbb{R}^{2}$ we have

$$
\mathcal{Z}_{i}=\left(\mathcal{Z}_{i L} \cap \mathcal{R}_{L}\right) \cup\left(\mathcal{Z}_{i C} \cap \mathcal{R}_{C}\right) \cup\left(\mathcal{Z}_{i R} \cap \mathcal{R}_{R}\right) .
$$

By continuity of $\dot{V}_{i}$, we have $\mathcal{Z}_{i L} \cap \mathcal{D}_{-1}=\mathcal{Z}_{i C} \cap \mathcal{D}_{-1}$, $\mathcal{Z}_{i R} \cap \mathcal{D}_{1}=\mathcal{Z}_{i C} \cap \mathcal{D}_{1}$.

Let us characterize the sets $\mathcal{Z}_{1 L}, \mathcal{Z}_{1 C}$ and $\mathcal{Z}_{1 R}$. According to (8), we have

$$
\begin{aligned}
\dot{V}_{1 L}(x)= & x^{\top} Q_{1} x-2 x^{\top} P_{1} B \\
& \quad+B^{\top} P_{1} Q_{1}^{-1} P_{1} B-B^{\top} P_{1} Q_{1}^{-1} P_{1} B \\
= & W_{1 L}(x)-d_{1}
\end{aligned}
$$

where $W_{1 L}(x):=\left(x-Q_{1}^{-1} P_{1} B\right)^{\top} Q_{1}\left(x-Q_{1}^{-1} P_{1} B\right)$ and $d_{1}:=B^{\top} P_{1} Q_{1}^{-1} P_{1} B$. From the above identity, the set $\mathcal{Z}_{1 L}=\left\{x \in \mathbb{R}^{2} \mid \dot{V}_{1 L}(x)=0\right\}=\left\{x \in \mathbb{R}^{2} \mid W_{1 L}(x)=d_{1}\right\}$ 
is an ellipse centred at $x_{c_{1}}:=Q_{1}^{-1} P_{1} B$.

Proceeding the same way for $\dot{V}_{1 R}(x)$, we obtain $\dot{V}_{1 R}(x)=$ $W_{1 R}(x)-d_{1}$ with $W_{1 R}(x):=\left(x+x_{c_{1}}\right)^{\top} Q_{1}\left(x+x_{c_{1}}\right)$ and the set $\mathcal{Z}_{1 R}=\left\{x \in \mathbb{R}^{2} \mid \dot{V}_{1 R}(x)=0\right\}=\left\{x \in \mathbb{R}^{2} \mid W_{1 R}(x)=\right.$ $\left.d_{1}\right\}$ is the image of $\mathcal{Z}_{1 L}$ by central symmetry, yielding an ellipse centred at $-x_{c_{1}}$.

Finally, let us characterize $\mathcal{Z}_{1 C}=\left\{x \in \mathbb{R}^{2} \mid \dot{V}_{1 C}(x)=\right.$ $0\}=\left\{x \in \mathbb{R}^{2} \mid x^{\top} Q_{1_{C L}} x=0\right\}$. From Lemma 2.2, the matrix $Q_{1_{C L}}$ is not sign definite and $\operatorname{det}\left(Q_{1_{C L}}\right)<0$. Thus the set $\mathcal{Z}_{1 C}$ consists of two lines passing through the origin. From $(7), Q_{1_{C L}(2,2)} \neq 0$ since $\left[\begin{array}{ll}0 & 1\end{array}\right] Q_{1_{C L}}\left[\begin{array}{l}0 \\ 1\end{array}\right]<0$. The solution to $x^{\top} Q_{1_{C L}} x=0$, subject to $x \in \mathcal{D}_{1}$ is

$$
\left[\begin{array}{c}
1 \\
\frac{-Q_{1_{C L}(1,2)}-\sqrt{-\operatorname{det}\left(Q_{1_{C L}}\right)}}{Q_{1_{C L}(2,2)}}
\end{array}\right],\left[\begin{array}{c}
1 \\
\frac{-Q_{1_{C L}(1,2)}+\sqrt{-\operatorname{det}\left(Q_{1_{C L}}\right)}}{Q_{1_{C L}(2,2)}}
\end{array}\right],
$$

from which we obtain the two lines defining $\mathcal{Z}_{1 C}$ and the coordinates of the intersection $\mathcal{Z}_{1 C} \cap \mathcal{D}_{1}$. The sets $\mathcal{Z}_{1 L}, \mathcal{Z}_{1 C}$ and $\mathcal{Z}_{1 R}$ are depicted in Figure 1. Using the

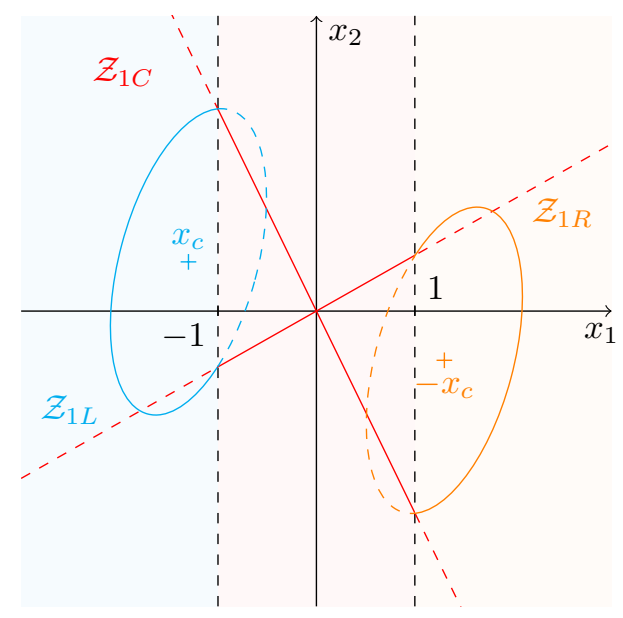

Figure 1: The sets $\mathcal{Z}_{1 L}, \mathcal{Z}_{1 C}$ and $\mathcal{Z}_{1 R}$.

central symmetry between $\mathcal{Z}_{1 L}$ and $\mathcal{Z}_{1 R}$ and the continuity property of $\dot{V}_{1}$, we obtain the sets where $\dot{V}_{1}(x)>0$ and $\dot{V}_{1}(x)<0$ as depicted in Figure 2.

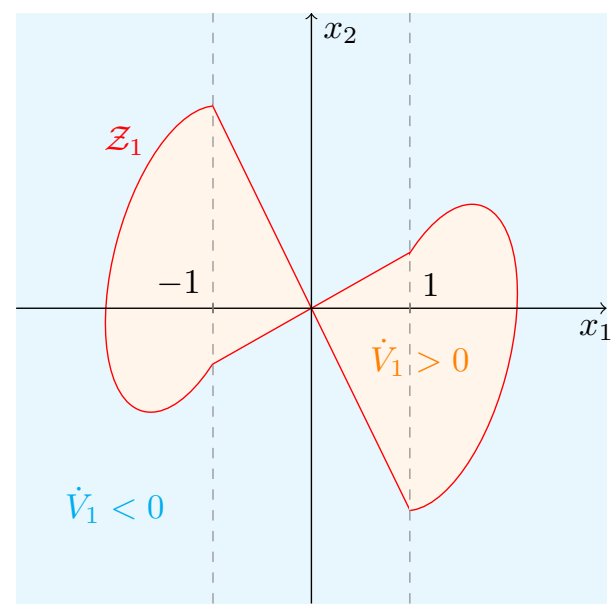

Figure 2: The partition of $\mathbb{R}^{2}$ based on the sign of $\dot{V}_{1}$.
We now study sets $\mathcal{Z}_{2 L}, \mathcal{Z}_{2 C}$ and $\mathcal{Z}_{2 R}$. Similar to $\dot{V}_{1 L}(x)$, we have $\dot{V}_{2 L}(x)=W_{2 L}(x)-d_{2}$ where $W_{2 L}(x):=\left(x-Q_{2}^{-1} P_{2} B\right)^{\top} Q_{2}\left(x-Q_{2}^{-1} P_{2} B\right)$ and $d_{2}:=B^{\top} P_{2} Q_{2}^{-1} P_{2} B$. Since $Q_{2}$ is not sign definite the set $\mathcal{Z}_{2 L}=\left\{x \in \mathbb{R}^{2} \mid \dot{V}_{2 L}(x)=0\right\}=\left\{x \in \mathbb{R}^{2} \mid W_{2 L}=d_{2}\right\}$ is an hyperbole centred at $x_{c_{2}}:=Q_{2}^{-1} P_{2} B$.

In the same way $\dot{V}_{2 R}(x)=W_{2 R}(x)-d_{2}$ with $W_{2 R}(x):=\left(x+x_{c_{2}}\right)^{\top} Q_{2}\left(x+x_{c_{2}}\right)$ and the set $\mathcal{Z}_{2 R}=\left\{x \in \mathbb{R}^{2} \mid \dot{V}_{2 R}(x)=0\right\}=\left\{x \in \mathbb{R}^{2} \mid W_{2 R}=d_{2}\right\}$ is the image of $\mathcal{Z}_{2 L}$ by central symmetry, yielding an hyperbole centred at $-x_{c_{2}}$.

According to Assumption 2.1, $Q_{2_{C L}}=A_{C L}^{\top} P_{2}+P_{2} A_{C L}>$ 0 . Hence $\forall x \in \mathcal{R}_{C} \backslash\{0\}, \quad \dot{V}_{2}(x)>0$ and $\mathcal{Z}_{2 C}=\{0\}$. In particular, by continuity of $\dot{V}_{2}$ we have $\dot{V}_{2}(x)=\dot{V}_{2 C}(x)=\dot{V}_{2 R}(x)>0, \quad \forall x \in \mathcal{D}_{1}$, $\dot{V}_{2}(x)=\dot{V}_{2 C}(x)=\dot{V}_{2 L}(x)>0, \quad \forall x \in \mathcal{D}_{-1}$. Thus, $\mathcal{D}_{1}$ and $\mathcal{D}_{-1}$ do not intersect the hyperboles $\mathcal{Z}_{2 L}, \mathcal{Z}_{2 R}$. The regions where $\dot{V}_{2}(x)>0$ and $\dot{V}_{2}(x)<0$ are depicted in Figure 3.

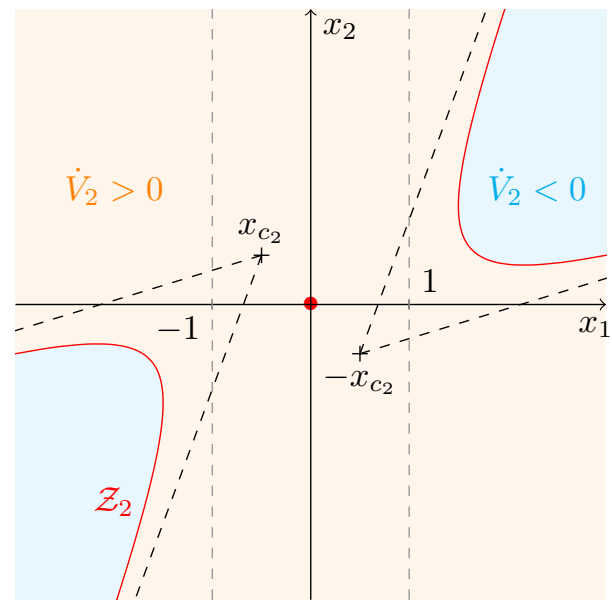

Figure 3: The partition of $\mathbb{R}^{2}$ based on the sign of $\dot{V}_{2}$.

Let us apply the above results to an example, that verifies Assumption 2.1.

Example 2.1 (Analysis): We consider system (3) with

$$
A=\left[\begin{array}{cc}
-1 & -1 \\
1 & 0
\end{array}\right] B=\left[\begin{array}{c}
2 \\
0.5
\end{array}\right] \text { which gives } A_{C L}=\left[\begin{array}{cc}
1 & -1 \\
1.5 & 0
\end{array}\right]
$$

With $Q_{1}=-I_{2}, Q_{2_{C L}}=I_{2}$ we solve (5) to obtain $P_{1}$ and $P_{2}$. The corresponding sets $\mathcal{Z}_{1}$ and $\mathcal{Z}_{2}$ are depicted in Figure 4.

\section{MAIN RESUlts}

We now provide a set of definitions required to introduce the main result of this section. To characterize the asymptotic behaviour of $\Phi\left(t, x_{0}\right), x_{0} \neq 0$ we introduce the following definitions.

Definition 3.1: The set $\mathcal{L}$ is said to be invariant with respect to system $\dot{x}=f(x)$ provided

$$
x_{0}=\Phi\left(0, x_{0}\right) \in \mathcal{L} \Rightarrow x(t)=\Phi\left(t, x_{0}\right) \in \mathcal{L}, \forall t \in \mathbb{R} .
$$




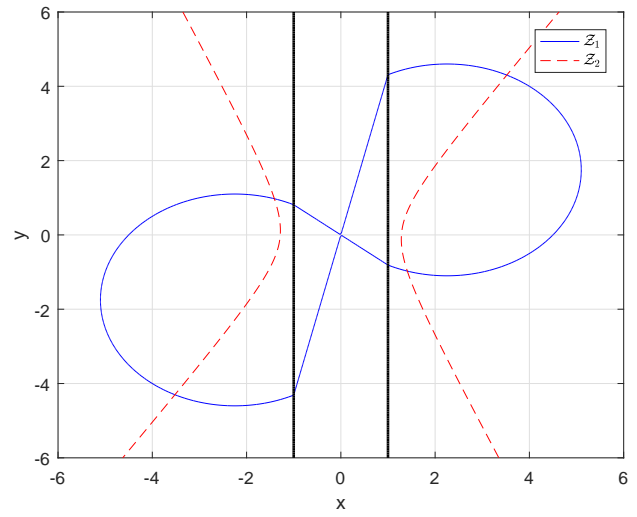

Figure 4: Sets $\mathcal{Z}_{1}$ and $\mathcal{Z}_{2}$ with $Q_{1}=-I_{2}, Q_{2_{C L}}=I_{2}$.

If the previous implication only holds for all $t \geq 0, \mathcal{L}$ is said to be positively invariant.

Definition 3.2: A set $\mathcal{L}$ is said to be finite-time attractive with respect to system $\dot{x}=f(x)$ provided for any trajectory $\Phi\left(t, x_{0}\right), x_{0} \in \mathbb{R}^{2}, \exists t^{*} \geq 0$ such that $\Phi\left(t, x_{0}\right) \in$ $\mathcal{L}, \forall t \geq t^{*}$.

Definition 3.3: A set $\mathcal{L}$ is said to be finite-time repulsive with respect to system $\dot{x}=f(x)$ provided that for any trajectory $\Phi\left(t, x_{0}\right), \exists t^{*} \geq 0$ such that $\Phi\left(t, x_{0}\right) \notin \mathcal{L}, \forall t \geq$ $t^{*}$.

Definition 3.4: A point $\mathrm{y}$ is an $\omega$-limit point of the trajectory $\Phi\left(t, x_{0}\right)$ if there exists a sequence $\left(t_{n}\right)_{n \in \mathbb{N}}$ in $\mathbb{R}$ so that $\lim _{n \rightarrow \infty} t_{n}=+\infty$ and $\lim _{n \rightarrow \infty} \Phi\left(t_{n}, x_{0}\right)=y$. The set of all $\omega$-limit point of $\Phi\left(t, x_{0}\right)$ is called $\omega$-limit set.

We now exploit the set $\mathcal{Z}_{1}$ to prove the existence of finite-time attractive sets.

Lemma 3.1: For any scalar $\beta>\max _{\mathcal{Z}_{1}} V_{1}(x)$, the set $\mathcal{E}\left(P_{1}, \beta\right)$ is finite-time attractive with respect to (3).

Proof: Since $\beta>\max _{\mathcal{Z}_{1}} V_{1}(x)$ we have $\mathcal{Z}_{1} \subset \mathcal{E}\left(P_{1}, \beta\right)$. From the definition of $\mathcal{Z}_{1}, \forall x \in \partial \mathcal{E}\left(P_{1}, \beta\right), \frac{\partial V_{1}}{\partial x} \dot{x}(t)=$ $\dot{V}_{1}(x(t))<0$, that is, in its boundary, the vector field in (3) points inwards the set $\mathcal{E}\left(P_{1}, \beta\right)$. Thus $\mathcal{E}\left(P_{1}, \beta\right)$ is positively invariant with respect to $(3)$.

Consider an arbitrary initial condition $x_{0}$ and denote $\beta_{0}=V_{1}\left(x_{0}\right)$. If $\beta_{0} \leq \beta$, we have $x_{0} \in \mathcal{E}\left(P_{1}, \beta\right)$ and finitetime attractiveness holds with $t^{*}=0$. If $\beta_{0}>\beta$, consider the set $\Gamma_{1}=\left\{x \mid \beta \leq V_{1}(x) \leq \beta_{0}\right\}$, which is a compact set containing $x_{0}$ and take $\delta=-\max _{\Gamma_{1}} \dot{V}_{1}(x)$ which verifies $\delta>0$ since $\dot{V}_{1}<0, \forall x \in \Gamma_{1}$. Now suppose that $\Phi\left(t, x_{0}\right) \in$ $\Gamma_{1} \forall t \geq 0$, that is, suppose $\beta \leq V_{1}\left(\Phi\left(t, x_{0}\right)\right) \leq \beta_{0} \forall t \geq 0$. Using $\delta$ defined above and the differentiability of $V_{1}$ we have that $\forall t \geq 0, \beta \leq V_{1}\left(\Phi\left(t, x_{0}\right)\right)$ and $V_{1}\left(\Phi\left(t, x_{0}\right)\right)=$ $V_{1}\left(\Phi\left(0, x_{0}\right)\right)+\int_{0}^{t} \dot{V}_{1}\left(\Phi\left(\theta, x_{0}\right)\right) d \theta=\beta_{0}+\int_{0}^{t} \dot{V}_{1}\left(\Phi\left(\theta, x_{0}\right)\right) d \theta \leq$ $\beta_{0}-\delta t$. Thus $\forall t>\frac{\beta_{0}-\beta}{\delta}$ the inequality above is no longer satisfied hence $\exists t^{*}$ such that $\Phi\left(t^{*}, x_{0}\right) \notin \Gamma_{1}$. Since $\mathcal{E}\left(P_{1}, \beta_{0}\right)$ is also a positively invariant set, the trajectory $\Phi\left(t, x_{0}\right)$ has to leave $\Gamma_{1}$ by its inner boundary that is $\partial \mathcal{E}\left(P_{1}, \beta\right)$ and we have $\Phi\left(t, x_{0}\right) \in \mathcal{E}\left(P_{1}, \beta\right) \forall t>t^{*}$. Thus, the set $\mathcal{E}\left(P_{1}, \beta\right)$ is finite-time attractive.

We now exploit the sets delimited by $\mathcal{Z}_{2}$ to obtain finite- time repulsive sets.

Lemma 3.2: For any scalar $\alpha<\min _{\mathcal{Z}_{2} \backslash\{0\}} V_{2}(x)$, the set $\mathcal{E}\left(P_{2}, \alpha\right) \backslash\{0\}$ is finite-time repulsive.

Proof: Since $\alpha<\min _{\mathcal{Z}_{2} \backslash\{0\}} V_{2}(x)$ we have $\mathcal{E}\left(P_{2}, \alpha\right) \cap \mathcal{Z}_{2}=$ $\{0\}$. From the definition of $\mathcal{Z}_{2}, \forall x \in \partial \mathcal{E}\left(P_{2}, \alpha\right), \frac{\partial V_{2}}{\partial x} \dot{x}(t)=$ $\dot{V}_{2}(x(t))>0$, that is, in its boundary, the vector field in (3) points outwards the set $\mathcal{E}\left(P_{2}, \alpha\right)$. Thus, $\mathcal{E}^{c}\left(P_{2}, \alpha\right):=$ $\mathbb{R}^{2} \backslash \mathcal{E}\left(P_{2}, \alpha\right)$ is positively invariant with respect to (3).

Consider an arbitrary initial condition $x_{0} \neq 0$ and denote $\alpha_{0}=V_{2}\left(x_{0}\right)$. If $\alpha_{0} \geq \alpha$, we have $x_{0} \in \mathcal{E}^{c}\left(P_{2}, \alpha\right)$ and finite-time repulsiveness holds with $t^{*}=0$. If $\alpha_{0} \leq \alpha$, consider the set $\Gamma_{2}=\left\{x \mid \alpha_{0} \leq V_{2}(x) \leq \alpha\right\}$, which is a compact set containing $x_{0}$. Take $\delta=\min _{\Gamma_{2}} \dot{V}_{2}(x)$ which verifies $\delta>0$ since $\dot{V}_{2}>0 \forall x \in \Gamma_{2}$, that is, suppose $\alpha_{0} \leq V_{2}\left(\Phi\left(t, x_{0}\right)\right) \leq \alpha \forall t \geq 0$. Using $\delta$ obtained above and the differentiability of $V_{2}$ we have $\forall t \geq 0, \alpha \geq V_{2}\left(\Phi\left(t, x_{0}\right)\right)$ and $V_{2}\left(\Phi\left(t, x_{0}\right)\right)=V_{2}\left(\Phi\left(0, x_{0}\right)\right)+$ $\int_{0}^{t} \dot{\bar{V}}_{2}\left(\Phi\left(\theta, x_{0}\right)\right) d \theta=\alpha_{0}+\int_{0}^{t} \dot{V}_{2}\left(\Phi\left(\theta, x_{0}\right)\right) d \theta \geq \alpha_{0}+\delta t$. Thus, $\forall t>\frac{\alpha-\alpha_{0}}{\delta}$ the inequality is no longer satisfied hence $\exists t^{*}$ such that $\Phi\left(t^{*}, x_{0}\right) \notin \Gamma_{2}$. Since $\mathcal{E}^{c}\left(P_{2}, \alpha_{0}\right)$ is also a positively invariant set, the trajectory $\Phi\left(t, x_{0}\right)$ has to leave $\Gamma_{2}$ by its outer boundary that is $\partial \mathcal{E}\left(P_{2}, \alpha\right)$ and we have $\Phi\left(t, x_{0}\right) \notin \mathcal{E}\left(P_{2}, \alpha\right) \forall t>t^{*}$. Thus, the set $\mathcal{E}\left(P_{2}, \alpha\right)$ is finitetime repulsive.

From the above results, we can obtain the following property.

Proposition 3.1: For any $x_{0} \neq 0$, the trajectory $\Phi\left(t, x_{0}\right)$, solution of (3) verifying Assumption 2.1 converges to a periodic orbit that encircles the origin.

Proof: From lemmas 3.1 and 3.2 there exists an finitetime attractive set $\mathcal{E}\left(P_{1}, \beta\right)$ and a finite-time repulsive set $\mathcal{E}\left(P_{2}, \alpha\right)$ with $\beta>\max _{\mathcal{Z}_{1}} V_{1}(x)$ and $\alpha<\min _{\mathcal{Z}_{2} \backslash\{0\}} V_{2}(x)$. Thus, $\forall x_{0} \in \mathbb{R}^{2} \backslash\{0\}, \exists t^{*}$ such that $\forall t \geq t^{*}, \Phi\left(t, x_{0}\right) \in \mathcal{E}\left(P_{1}, \beta\right)$, $\Phi\left(t, x_{0}\right) \notin \mathcal{E}\left(P_{2}, \alpha\right)$. That is, $\forall t \geq t^{*}, \Phi\left(t, x_{0}\right) \in \mathcal{R}:=$ $\mathcal{E}\left(P_{1}, \beta\right) \backslash \mathcal{E}\left(P_{2}, \alpha\right)$. The set $\mathcal{R}$ is finite-time attractive and does not contain the origin, which is the only equilibrium point of (3) (from Lemma 2.1). Hence, from [5, Theorem 7.1 on p.290], the $\omega$-limit set $L^{+}$of $\Phi\left(t, x_{0}\right)$ is a periodic orbit. Since inside any periodic orbit of a planar system there must exists at least one equilibrium point (see [5, Corollary 7.1 on p.299]), we conclude that $L^{+}$encircles the origin.

Remark 3.1: Since $L^{+} \in \mathcal{R}$ and $L^{+}$encircles the origin, the set inclusion $\mathcal{E}\left(P_{2}, \alpha\right) \subset \mathcal{E}\left(P_{1}, \beta\right)$ must hold.

\section{A. Systems with dead-zone}

Consider the system with unit dead-zone function $\dot{\bar{x}}=$ $\bar{A} \bar{x}+\bar{B} \mathrm{dz}(\bar{K} \bar{x})$. Following Remark 2.1, the above system can be written as (1), that is $\dot{\bar{x}}=(\bar{A}+\bar{B} \bar{K}) x-\bar{B} \operatorname{sat}(\bar{K} x)$. Thus, Assumption 2.1 applied to the above system becomes

Assumption 3.1 (Deadzone systems): $-A$ and $A_{C L}$ are Hurwitz matrices.

In view of Proposition 3.1, for open loop unstable systems (that is $-A$ is Hurwitz) with dead-zone on its input, a 
stabilising feedback gain $K$ (that is $A+B K$ is Hurwitz) guarantees that any trajectory with $x_{0} \neq 0$ converges to a periodic orbit.

\section{Estimation OF PERIODIC ORBITS}

In the previous section we showed that the set containing all the periodic trajectories is a ring delimited by two ellipsoids. This section presents results allowing to compute a ring containing the periodic trajectories. That is, to compute ellipsoids $\mathcal{E}\left(P_{1}, \beta\right)$ and $\mathcal{E}\left(P_{2}, \alpha\right)$ by letting either $P_{1}$ and $P_{2}$ or $\alpha$ and $\beta$ to be unknowns. We propose algorithms that allow for the 1) minimisation of the outer set $\mathcal{E}\left(P_{1}, \beta\right)$ for $P_{1}$ or $\beta$ given; 2$)$ maximisation of the inner set $\mathcal{E}\left(P_{2}, \alpha\right)$ for $P_{2}$ or $\alpha$ given. The lemma below, that relies on the symmetry of both $\mathcal{E}\left(P_{1}, \beta\right)$ and $\mathcal{Z}_{1}$, (depicted in Figure 2), and the convexity of $\mathcal{E}\left(P_{1}, \beta\right)$, to establish conditions that ensure the set inclusion

$$
\mathcal{Z}_{1} \subset \mathcal{E}\left(P_{1}, \beta\right) .
$$

Lemma 4.1: The set inclusion (9) holds if and only if

$$
\mathcal{H}_{R}:=\left\{x \in \mathbb{R}^{2} \mid K x \geq 1, \dot{V}_{1 R} \geq 0\right\} \subset \mathcal{E}\left(P_{1}, \beta\right) .
$$

Proof: From the symmetry with respect to the origin of $\mathcal{E}\left(P_{1}, \beta\right)$, we have that (10) implies $\mathcal{H}_{L}:=$ $\left\{x \in \mathbb{R}^{2} \mid K x \leq-1, \dot{V}_{1 L} \geq 0\right\} \subset \mathcal{E}\left(P_{1}, \beta\right)$. Now, from the convexity of $\mathcal{E}\left(P_{1}, \beta\right)$, we conclude $\operatorname{Conv}\left(\mathcal{H}_{R} \cup \mathcal{H}_{L}\right) \subset$ $\mathcal{E}\left(P_{1}, \beta\right)$. Since at the same time $\mathcal{Z}_{1} \subset \operatorname{Conv}\left(\mathcal{H}_{R} \cup \mathcal{H}_{L}\right)$, by transitivity we obtain $\mathcal{Z}_{1} \subset \mathcal{E}\left(P_{1}, \beta\right)$.

A sufficient condition to verify the inclusion (10) is formulated with the $S$-procedure and is given in the proposition below.

Proposition 4.1: If there exist two positives scalars $\tau_{1} \geq$ $0, \tau_{2} \geq 0$ such that the matrix inequality

$$
\begin{aligned}
M_{1}\left(\tau_{1}, \tau_{2}\right):= & {\left[\begin{array}{cc}
-P_{1} & 0 \\
0 & \beta
\end{array}\right]+\tau_{1}\left[\begin{array}{cc}
-\left(A^{\top} P_{1}+P_{1} A\right) & -P_{1} B \\
-B^{\top} P_{1} & 0
\end{array}\right] } \\
& +\tau_{2}\left[\begin{array}{cc}
0 & -\frac{1}{2} K^{\top} \\
-\frac{1}{2} K & 1
\end{array}\right] \geq 0
\end{aligned}
$$

holds then inclusion (10) holds.

The proof is a straightforward application of the $S$ procedure [11],[1, Chapter 2.6.3].

Next we present necessary and sufficient condition for the following set inclusion

$$
\mathcal{E}\left(P_{2}, \alpha\right) \subset\left\{x \mid \dot{V}_{2}(x)>0\right\} \cup\{0\},
$$

to hold. Notice that this inclusion implies $\mathcal{E}\left(P_{2}, \alpha\right) \cap \mathcal{Z}_{2}=$ $\{0\}$ (refer to Figure 3 for a depiction of the sets).

Lemma 4.2: The set inclusion $\mathcal{E}\left(P_{2}, \alpha\right)$ $\left\{x \mid \dot{V}_{2}(x)>0\right\} \cup\{0\}$ holds if and only if

$$
\begin{gathered}
\mathcal{F}_{R}:=\left\{x \in \mathbb{R}^{2} \mid K x \geq 1, x^{\top} P_{2} x \leq \alpha\right\} \\
\subset\left\{x \in \mathbb{R}^{2} \mid \dot{V}_{2}(x)>0\right\} .
\end{gathered}
$$

Proof: $\quad$ Define $\quad \mathcal{F}_{L} \quad:=\quad\left\{x \in \mathbb{R}^{2}\right.$ $\left.\mid K x \leq-1, x^{\top} P_{2} x \leq \alpha\right\}$. From the symmetry of $\mathcal{E}\left(P_{2}, \alpha\right)$ with respect to the origin, we have that if (13) holds then $\mathcal{F}_{L} \subset\left\{x \in \mathbb{R}^{2} \mid \dot{V}_{2}(x)>0\right\}$ also holds. The set $\mathcal{F}_{C}:=\left\{x \in \mathbb{R}^{2} \mid-1<K x<1\right\}$ verifies $\mathcal{F}_{C} \subset\left\{x \in \mathbb{R}^{2} \mid \dot{V}_{2}(x)>0\right\} \cup\{0\}$. Thus, since $\mathcal{E}\left(P_{2}, \alpha\right)=\mathcal{F}_{L} \cup \mathcal{F}_{C} \cup \mathcal{F}_{R}$, if (13) holds we have $\mathcal{E}\left(P_{2}, \alpha\right) \subset\left\{x \mid \dot{V}_{2}(x)>0\right\} \cup\{0\}$.

The proposition below is a sufficient condition to verify the inclusion (13).

Proposition 4.2: If there exist two scalars $\tau_{1} \geq 0, \tau_{2} \geq 0$ such that

$$
\begin{aligned}
M_{2}\left(\tau_{1}, \tau_{2}\right):= & {\left[\begin{array}{cc}
A^{\top} P_{2}+P_{2} A & P_{2} B \\
B^{\top} P_{2} & 0
\end{array}\right]+\tau_{1}\left[\begin{array}{cc}
P_{2} & 0 \\
0 & -\alpha
\end{array}\right] } \\
& +\tau_{2}\left[\begin{array}{cc}
0 & -\frac{1}{2} K^{\top} \\
-\frac{1}{2} K & 1
\end{array}\right] \geq 0
\end{aligned}
$$

holds, then inclusion (13) holds.

The conditions for the set inclusions (9), (12) to hold, established by propositions 4.1, 4.2 are expressed in terms of matrix inequalities. Therefore they are convenient for the formulation of numerical procedures to compute estimates of the set containing the periodic orbit. Indeed, these inequalities can be used as constraints of optimization problems, namely semi-definite programs (SDP). In case these constraints have an affine dependence on the unknowns, the feasible set is convex. When associated to a linear objective function, a convex optimization problem is cast and its solution can be obtained using freely available software [6], [8]. In the remaining of the section we exploit the set inclusion inequalities (9), (12) to formulate SDP programs thus providing estimates of the periodic trajectories.

We first provide a solution for the following problems for a saturating system given a feedback gain satisfying Assumption 2.1.

Problem 4.1 (Computation of the outer estimate): For $P_{1}$ satisfying (5) for a given $Q_{1}$, compute the smallest scalar $\beta$ satisfying (9).

Based on the condition in Propositions 4.1 we propose the following (convex) SDP to solve the above problem

$$
\underset{\beta, \tau_{1}, \tau_{2}}{\operatorname{minimise}} \beta \text { subject to } M_{1} \geq 0, \beta \geq 0, \tau_{1} \geq 0, \tau_{2} \geq 0 \text {. }
$$

Problem 4.2 (Computation of inner estimate): For $P_{2}$ satisfying (5) for a given $Q_{2_{C L}}$, compute the largest scalar $\alpha$ satisfying (12).

Based on the condition in Propositions 4.2 we propose the following (convex) SDP to solve the above problem

$$
\underset{\alpha, \tau_{1}, \tau_{2}}{\operatorname{maximise}} \alpha \text { subject to } M_{2} \geq 0, \alpha \geq 0, \tau_{1} \geq 0, \tau_{2} \geq 0 \text {. }
$$

Consider again Example 2.1. We solve the above SDPs with $Q_{1}=-I_{2}$ and $Q_{2_{C L}}=I_{2}$ to obtain inner and outer estimates (depicted in green in Figure 5). A periodic trajectory is also depicted to illustrate the containment in the ring delimited by the two ellipsoids.

Note that the computed estimates are optimal for a given $P_{i}$, not necessarily corresponding to the closest inner and outer ellipsoids to the periodic trajectories. Therefore, to obtain better estimates, we consider matrices $P_{i}$, related to the ellipsoid shape, as variables. Letting $P_{i}$ vary requires the corresponding (variables) $Q_{1}$ and $Q_{2_{C L}}$ to satisfy 


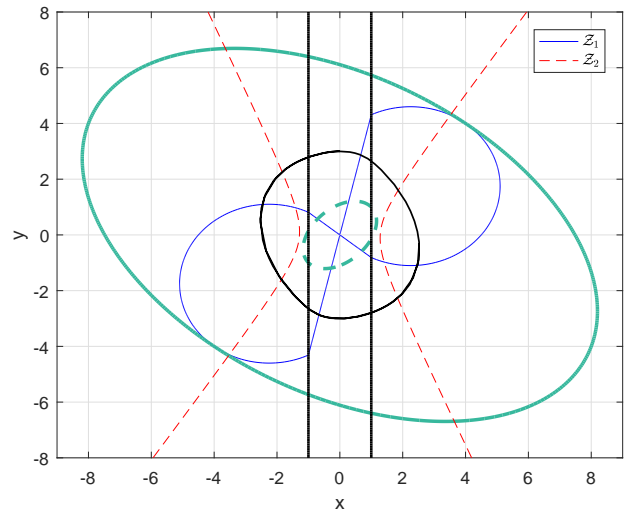

Figure 5: Outer and inner estimates with $Q_{1}=-I_{2}$, $Q_{2_{C L}}=I_{2}$ for Example 2.1.

$Q_{1} \in \mathbb{S}_{<0}$ and $Q_{2_{C L}} \in \mathbb{S}_{>0}$. Such a requirement imposes the inequalities

$$
\begin{array}{r}
A^{\top} P_{1}+P_{1} A<0 \\
A_{C L}^{\top} P_{2}+P_{2} A_{C L}>0 .
\end{array}
$$

in the formulation of the optimization problem. Whenever $P_{1}\left(P_{2}\right)$ is an optimization variable we fix the scalar $\beta$ $(\alpha)$ defining the set $\mathcal{E}\left(P_{1}, \beta\right)\left(\mathcal{E}\left(P_{2}, \alpha\right)\right)$. We now propose optimization-based solutions to the problems

Problem 4.3: Given $\beta>0$, compute a matrix $P_{1}$ such that (9) and (15) hold and $\mathcal{E}\left(P_{1}, \beta\right)$ is as close as possible to the periodic orbit.

Problem 4.4: Given $\alpha>0$, compute a matrix $P_{2}$ such that (12) and (16) hold and $\mathcal{E}\left(P_{2}, \alpha\right)$ is as close as possible to the periodic orbit.

To set an SDP to solve the above problems we define a linear function on the decisions variables related to the distance to the periodic trajectories. A possible criteria is the trace of the matrix $P_{i}$ which is adopted below. Note that whenever $P_{i}$ is a variable, to obtain a convex optimization problem using (11) (or (14)) parameter $\tau_{1}$ has to be fixed. Thus, to solve problems 4.3 and 4.4 , we perform a line search on parameter $\tau_{1}$ by solving the SDPs for a fixed values of $\tau_{1}$

$$
\begin{aligned}
& \underset{P_{1}, \tau_{2}}{\operatorname{maximise}} \operatorname{trace}\left(P_{1}\right) \text { subject to }(11), P_{1}>0, \tau_{2} \geq 0, \\
& \underset{P_{2}, \tau_{2}}{\operatorname{minimise}} \operatorname{trace}\left(P_{2}\right) \text { subject to }(14),(16), P_{2}>0, \tau_{2} \geq 0 .
\end{aligned}
$$

We consider again Example 2.1. We let $P_{1}\left(P_{2}\right)$ be a variable and we solve Problem 4.3 (4.4) by performing a line search on $\tau_{1}$. The results are depicted in Figure 6 .

\section{Conclusion}

For planar saturating systems we established conditions on the system matrix for the existence of periodic trajectories. Moreover we have characterized a set defined by two ellipsoids which contains periodic trajectories. A parametrization of such a set in terms of matrix inequalities has allowed us to estimate sets containing the periodic trajectories by solving convex optimization problems. We

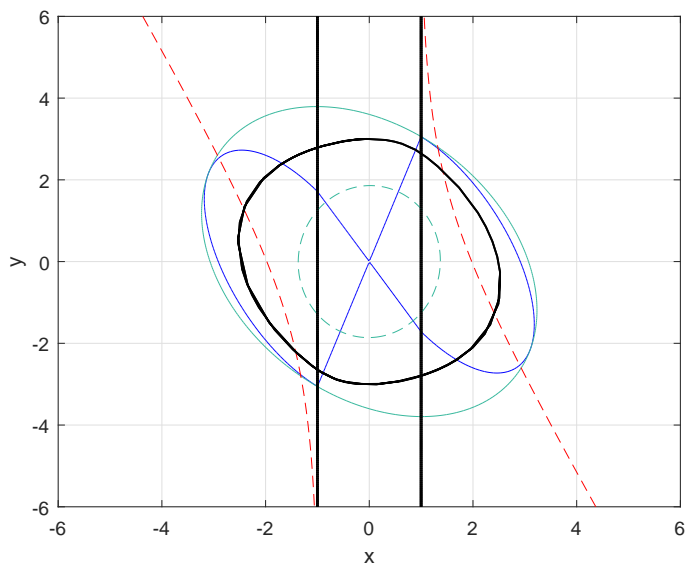

Figure 6: Outer and inner estimates of example 2.1 with $Q_{1}=-I_{2}$ for $P_{1}$ variable.

are currently developing strategies for feedback design that generate periodic trajectories.

\section{REFERENCES}

[1] S. Boyd, L. El Ghaoui, E. Feron, and V. Balakrishnan. Linear matrix inequalities in system and control theory, volume 23 of SIAM Studies in Applied Mathematics. SIAM, 1994.

[2] F. Ferrante, F. Gouaisbaut, and S. Tarbouriech. Stabilization of continuous-time linear systems subject to input quantization. Automatica, 58:167-172, 2015.

[3] T. Hu and Z. Lin. Control Systems with Actuator Saturation: Analysis and Design. Birkhäuser Boston, 2001.

[4] T. Hu, T. Thibodeau, and A. R. Teel. A unified Lyapunov approach to analysis of oscillations and stability for systems with piecewise linear elements. IEEE Transactions on Automatic Control, 55(12):2864-2869, 2010.

[5] Hassan K Khalil. Noninear Systems. Prentice-Hall, New Jersey, 2 edition, 1996

[6] J. Löfberg. Yalmip : A toolbox for modeling and optimization in matlab. In In Proceedings of the CACSD Conference, Taipei, Taiwan, 2004.

[7] R. Ouyang, V. Andrieu, and B. Jayawardhana. On the characterization of the Duhem hysteresis operator with clockwise input-output dynamics. Systems and Control Letters, 62(3):286-293, 2013

[8] Jos F. Sturm. Using SeDuMi 1.02, a matlab toolbox for optimization over symmetric cones, 1998.

[9] S. Tarbouriech, I. Queinnec, and C. Prieur. Stability analysis and stabilization of systems with input backlash. IEEE Transactions on Automatic Control, 59(2):488-494, 2014.

[10] A.R. Teel. Global stabilization and restricted tracking for multiple integrators with bounded controls. Systems and Control Letters, 18:165-171, 1992.

[11] V. A. Yakubovich. The S-procedure in non-linear control theory. Vestnik Leningrad Univ. Math., 4:73-93, 1977. 